\title{
Re-Sheathing the Scimitar Syndrome
}

\author{
Michael Michail, Kaleab N. Asrress, Abhishek Joshi, John A. Giles, \\ Muhammed Z. Khawaja, Eric T. McWilliams \\ Department of Cardiology, Conquest Hospital, Hastings, UK \\ Email: eric.mcwilliams@gmail.com
}

Received November 10, 2012; revised December 12, 2012; accepted December 20, 2012

\begin{abstract}
Scimitar Syndrome is a congenital condition resulting in anomalous pulmonary venous drainage from the lung into the inferior vena cava. It is often associated with cardiac and pulmonary abnormalities, both which are determinants in the severity and timing of presentation. True incidence of this condition is unknown and likely to be significantly underestimated as many patients remain asymptomatic through adult life. The increase in availability and use of medical imaging suggests that more asymptomatic and subacute cases will be detected. The management in those with the delayed presentation of this condition is debated, however the mainstay of therapy remains medical.
\end{abstract}

Keywords: Adult-Onset Scimitar Syndrome; Multidetector Computed Tomography

\section{Case Report}

A fifty-year-old lady presented with progressive exertional dyspnoea in absence of significant past medical history. Clinical examination was normal. Twelve lead electrocardiograms showed non-specific changes. Biomarkers including full blood count, cardiac troponin-T, and D-dimers were normal. Plain chest radiograph showed abnormal vascular markings in the right lower zone suspicious of a scimitar vein (SV) (Figure 1(A)). 64-slice multidetector computed tomography showed partial anomalous pulmonary venous drainage of the entire right lung to the inferior vena cava (IVC) below the right atrial-IVC junction (Figures 1(B)-(D)), confirming scimitar syndrome (SS). Cardiac catheterisation confirmed the presence of the SV, and found a left-to-right shunt with $\mathrm{Q}_{\mathrm{p}}: \mathrm{Q}_{\mathrm{s}}$ of 2.1:1.

\section{Scimitar Syndrome}

SS is a rare congenital abnormality, characterised by anomalous pulmonary venous drainage of the right, or less frequently, the left lung, into the inferior vena cava. The true incidence is unknown. The syndrome is named after the Turkish sword "scimitar" describing the radiographic appearance of the anomalous vein.

SS has two distinct presentations. The "infant" form is associated with other congenital abnormalities and greater mortality. Clinical features may include failure to thrive, tachypnoea, cardiac failure, recurrent chest infections and cyanosis [1]. Medical therapy is used to alleviate symptoms in infants, however the presence of pulmonary hypertension warrants prompt surgical correc- tion.

The second form presents in children and adults. Cardiac and pulmonary abnormalities are associated, the former more accountable for the severity of symptoms. Cardiac abnormalities include dextrocardia, atrial and ventricular septal defects, patent ductus arteriosus and pulmonary vein stenosis. Pulmonary abnormalities vary but commonly include right lung hypoplasia and pulmonary sequestration. In two thirds of patients, the SV provides drainage for the entire right lung.

Delayed presentation in the adult group is the result of fewer cardiopulmonary anomalies. Patients present with exertional dyspnoea and recurrent chest infections, more commonly involving the right lung. Electrocardiogram may find atrial fibrillation, right ventricular hypertrophy or right bundle branch block. Pulmonary function tests demonstrate mild deficits in vital capacity [2]. Computed tomography is increasingly used to provide $3 \mathrm{D}$ images to guide further treatment [3].

\section{Treatment}

The conventional indications for corrective surgery in adults with SS are a shunt of greater than 50\% (a ratio of 2:1), or pulmonary sequestration with recurrent pulmonary infection. These indications arose from a small, single centre study $(n=6)$ [4].

Corrective surgery attempts to reduce left-to-right shunt by diverting the flow of SV into systemic circulation. Two main approaches have been used: First, re-implantation of the SV into the left atrium or, second, to use a baffle to redirect flow across the right atrium through 

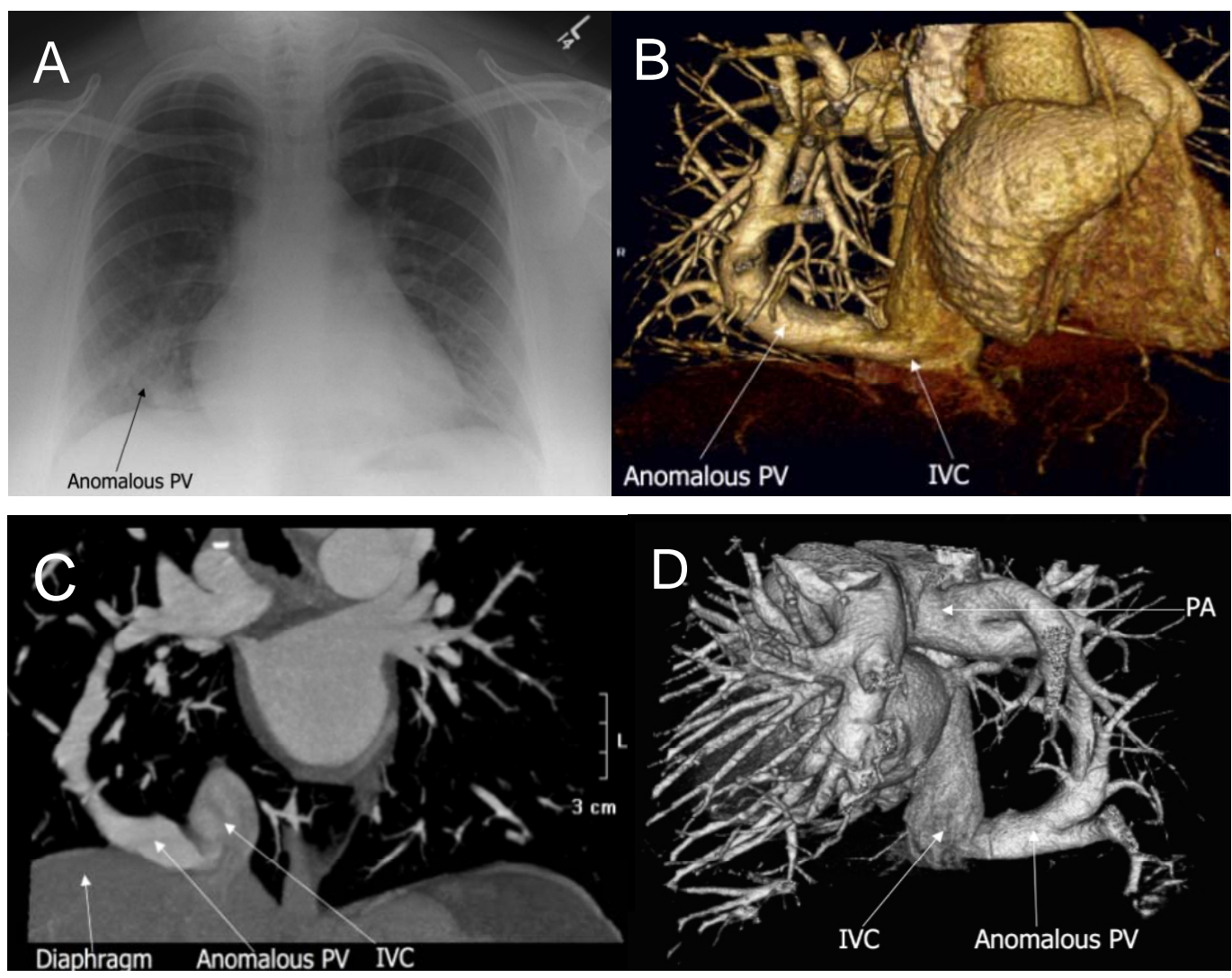

Figure 1. Chest radiograph (A) and 64-slice multidetector computed tomography (B-D). IVC indicates inferior vena cava; PA, pulmonary artery and PV, pulmonary vein.

an existing or created ASD. Both require cardiopulmonary bypass. Post-operative complications include catastrophic pulmonary vein occlusion requiring re-operation or occlusion of the baffle.

The largest, multi-centre series $(n=122)$ to report surgical outcomes in adult SS found 28 patients with pulmonary hypertension and 22 had left-to-right shunt greater than 50\%. Of those operated (37) 12 had "good outcomes” compared with 79 of 85 not undergoing surgical correction [2]. A single, small comparison study has favoured baffle procedures over direct vein implantation [5].

\section{Conclusion}

SS is a rare, congenital, cardiopulmonary abnormality that has two distinct clinical presentations. Practitioners are unlikely to see more than one in an entire career, but the increase in use of three-dimensional imaging mean more sub-acute or asymptomatic "adult" cases may be found. It is therefore important to note that, in most cases, surgery is not required. The initial indications for surgery in adults should be revisited in the light of advances in anaesthesia, operative and interventional techniques, and the medical management of pulmonary hypertension.

\section{REFERENCES}

[1] U. Gudjonsson and J. W. Brown, "Scimitar Syndrome," Seminars in Thoracic and Cardiovascular Surgery: Pedatric Cardiac Surgery Annual, Vol. 9, No. 1, 2006, pp. 5662. doi:10.1053/j.pcsu.2006.02.011

[2] C. Dupuis, L. A. Charaf, G. M. Breviere, P. Abou, M. Remy-Jardin and G. Helmius, "The 'Adult' Form of the Scimitar Syndrome,” American Journal of Cardiology, Vol. 70, No. 4, 1992, pp. 502-507. doi:10.1016/0002-9149(92)91198-D

[3] T. Inoue, M. Ichihara, T. Uchida, Y. Sakai, T. Hayashi and S. Morooka, "Three-Dimensional Computed Tomography Showing Partial Anomalous Pulmonary Venous Connection Complicated by the Scimitar Syndrome," Circulation, Vol. 105, No. 5, 2002, p. 663. doi:10.1161/hc0502.101512

[4] M. Honey, “Anomalous Pulmonary Venous Drainage of Right Lung to Inferior Vena Cava ('Scimitar Syndrome'): Clinical Spectrum in Older Patients and Role of Surgery," QJM: An International Journal of Medicine, Vol. 46, No. 184, 1977, pp. 463-483.

[5] V. L. Vida, S. Speggiorin, M. A. Padalino, et al., "The Scimitar Syndrome: An Italian Multicenter Study," The Annals of Thoracic Surgery, Vol. 88, No. 2, 2009, pp. 440-444. doi:10.1016/j.athoracsur.2009.04.099 